\title{
But Why Two Integrative Conscious Brains?
}

\author{
Ahmad Yousef ${ }^{1}$ \\ ${ }^{1}$ School of Computational Science and Engineering, McMaster University, Hamilton, Ontario, Canada \\ *Correspondence: mohamas2@mcmaster.ca
}

\begin{abstract}
In this synoptic article, we provide evidential grounds that humans have two integrative conscious brains, a central conscious brain, and a peripheral one. The awareness created by the aforementioned brains are substantially different; however, they are normally functioning in an integrative way.
\end{abstract}

\section{Fulsome Discussion}

We showed that the human visual awareness has spacetime continuum, and spatial attention stretches that 'conscious spacetime continuum' causing dilation to the time and the size of the moving objects (see reference 1). Neuropsychologically speaking, it's the central perception that dilates time and length when compared with the peripheral perception. Distinctions of the time passage between the retinal centralis versus the retinal peripheries, however, were indirectly discussed "perceptually" by Traschütz etal. (see reference 2), and "physiologically" by Sinha et al. (see reference 3).

To validate theory of the two integrative conscious brains which is the neural basis of the aforementioned continuum, we have not only showed that the humans should have two distinct medial temporal complexes (see reference 4), as well as two distinct V4 areas (see reference 1); but the peripheral brain has so special characteristic, namely, it generates the illusory motion reversals (see references 5).

In further studies, we illustrate that the auditory awareness is also embedded in the theory of two integrative conscious brains, see reference 6 ; and we suggest that the other sensory systems such as tactile and olfactory would comply to the theory.

In the theory of the two integrative conscious brains, there are three optional operations where the central and the peripheral conscious brains can work collectively, see motion induced colours fusion illusion; illustratively, see the micro-rivalry in rotating snakes illusion and spatial wrapping; or one of these brains will be on while the other is off. Otherwise, two or more operations may occur simultaneously or sequentially. To show the difference between "collectively" and "illustratively"; we have to understand that the detachment between the background and the arrows in the stimuli given in (reference 7) is due to simultaneous feeds from the two conscious brains to build up the final awareness; however, when the two conscious brains would like to illustrate their spatiotemporal discrepancies, then the brain works illustratively, see reference 8 and 9 .

So far, we investigated basic geometrical components of human vision; and thus, some cognitive scientists could remain skeptical about the solidarity of our theory; investigating complex conscious task, for instance face recognition, is therefore seen to be a must. Astoundingly, we have proven that the fusiform face area shall have two distinct (central and peripheral) regions, see reference 10; and we were able to deactivate its peripheral area through a psychophysical trick that shall restrict the amount of oxygenated hemoglobin red blood cells from accessing the peripheral region, see reference 11 .

But what are the governors of the rivalrous processes? Inceptionally speaking, the dorsolateral prefrontal cortex seems to play a key role in switching the rivalrous perceptions generated by the two conscious brains; and this suggestion is a result of the fact that deep breathing and voluntary hand movements can both control the visual motion rivalry too effectively and in timely fashion, see reference 12 , and 13 . We suggested that it's the dorsolateral prefrontal cortex because it the highest cortical area for decision making; namely, it could order either the motor cortex to execute hand movement or the olfactory cortex to perform deep breathing. But the aforementioned neurological suggestion is not sufficient to identify the actual governor of the two conscious brains theory,

because of some special cases, for example; when human subjects spatial attend to one side of the stimulus given in (reference 1) while the stimulus subtends an extremely small degree visual angle; their attention still able to stretch the conscious spacetime continuum; and this observation jeopardizes our theory, because the entire stimulus is covered by the foveal region, unless 'the visual entanglement' process is found to be true. But what is the visual entanglement process? It is a process that assumes that the visual awareness resides in extra physical dimensions whereas the human brain projects its 'computations of synchronicity' to these dimensions through specialized inhibitory neurons. In another word, the entanglement process assumes that attention triggers these inhibitory neurons to emit particles to the extra physical dimensions, and these particles have to travel faster than light to govern the photons, namely, to create entanglements between the visual stimulus and its corresponding photoreceptors. Notice that these particles are only permitted to travel through extra physical dimensions, otherwise the laws of physics will be violated; for more details, see references 14, 15, and 16. In several binocular rivalry experiments achieved in our laboratory, we had proven that the rivalry can be stopped indefinitely through voluntary blinks, hand movements, as well as through involuntary foveal attention, see references 17,18 , and 19; also see the deep breathing effect but in a few mediative subjects in reference 20. Because of the possibility of the biological impossibilities to explain these indefinite stoppages, see reference 16 , and because there is no fulsome neurophysiological explanations for several visual phenomenon, for instance, the invisible gorilla, the attentional blinks, and the actual generator of visual imagery; and because of the fact that our mathematical 
description for tachyonic dynamics has established theoretical grounds in physics; we think that the presumed "visual entanglements" to be true. But we have to cautious about the fact that in the visual imagery scenarios, unless the external environment is supportive enough; the two entangled tachyons will travel exclusively together to the retina.

The two integrative conscious brains theory may fit all of the perceptual visual experiences, including but not limited to the binocular rivalry phenomena. In another word, the binocular rivalry is mostly a rivalry between the central brain 'neurally connected to the right eye' versus the peripheral brain 'but connected to the left eye'. Ultimate dominance or mixed percepts happen however when both of the two eyes' retinal centralis, or both of the two eyes' peripheries try to rival their images; namely, when the rivalry of the two conscious brains fails; either ultimate dominance or mixed percepts will prevail. The previous statement, however, requires confirmations of the tachyonic dynamics which will be offered in further conveyance.

In case of the readers are not following our previous work, we here provide a simplified theoretical proof of the physical existence of tachyons according to the well-established theoretical physics: Imagine the Laser Interferometer Gravitational-Wave Observatory 'LIGO system' but with extremely short two tubes, namely, the tubes' length has to be the laser's wavelength; the LIGO detector will therefore detect a zero; and this seriously violates the energy conservation principle. To resolve the aforementioned violation; we suggested that the energy were conveyed to extra physical dimensions, but since the super-string theory assumes that these extra dimensions are too small; thus, the momentum of the particles travelling through these dimensions have to be way smaller than the photons. But we have to be attentive here, because if these particles' momentum have to be way smaller, then their speed must be way faster; otherwise, the energy conservation principle will be again violated, see the following energy equations for better illustration:

$E_{\text {Before destructive interference }}=E_{\text {After destructive interference }}$

$$
x=\operatorname{det}\left(P_{\text {photon }} \times C\right)=w=\operatorname{det}\left(P_{\text {tachyon }} \times Y\right)
$$

Where the dot product of vectors $x$, and $w \rightarrow 0 ; C$ and $Y$ are the speeds of light, and tachyon respectively, and $P$ is the momentum; but the readers have to be again too careful that these are just mathematical illustrations; but our mathematical description for the tachyonic dynamics will be provided in a further notice. But it's very notable that the scientists had been searching for the "unknown" particles in incorrect manners; to understand the reasons, let's see the Planck's equation:

Energy $=$ Planck's constant $\times$ Frequency

But remember that because the LIGO detector shall detect a zero according to our thought experiment; therefore, the resultant particles will have zero value of the frequency; namely, the resultants' wavelength will be infinite. This suggests that scientists had been looking into the wrong planes and dimensions, namely, the Large Hadron Collider 'LHC system' will never be able to directly detect tachyons, unless their dynamics are considered in the detection design. The theory of two integrative conscious brains, however, considers fulsome version of the tachyonic dynamics expressed as 'the mathematical description for tachyonic dynamics.'
Within that description, several dynamics of physical phenomena were explained, for example the quantum tunneling, the quantum entanglement, as well as the 'generic gravity'; whereas graviton are assumed to be a special version of the tachyon.

The mathematical description for tachyonic dynamics, however, is not sure of how to design detectors for tachyons; we however suggested these detectors are already there in the human retina, but they're remained undiscovered. To discover those receptors, a retinal surgery for a macaque monkey was suggested in reference 16. Finally, the readers has to be cautious about our statements, we're not saying that the visual entanglement but not anything else generates the visual experiences; however, we indeed had clearly stated that there are networked parameters that are all working together to achieve phenomenal human visual awareness, see references 5, 21 and 22. These parameters include, neural and cardiac activities, eye movements, blinks, pupil size variations, the dynamics between the pupil and the suspensory ligaments of the lens; and surely, the visual entanglement. These parameters are all correlated; but the main governor of the awareness, we believe, is the visual entanglement!

\section{Summary}

To sum, we have to state it clearly that we believe that the human visual and other sensory awareness can be fully explained through the theory of the two integrative conscious brains as long as the tachyonic transmissions are proven to be true.

\section{Transactional References}

[1] Yousef, 2019. "Spatial Attention Dilates Time and Length." PsyArXiv. doi:10.31234/osf.io/qazj9.

[2] Traschütz etal., 2012. Speed change detection in foveal and peripheral vision. Vision Research.

[3] Sinha, etal. (2017). Cellular and circuit mechanisms shaping the perceptual properties of the primate fovea. Cell 168, 413-426.

[4] Yousef, 2020. "Two Distinct Human Medial Temporal Complexes." PsyArXiv. doi:10.31234/osf.io/8dzkp.

[5] Yousef, 2019. "Retinal Peripheries Generate Illusory Motion Reversals." PsyArXiv. doi:10.31234/osf.io/gfmpe.

[6] Yousef, 2019. "Distinctions Between Central and Peripheral Hearing." PsyArXiv. doi:10.31234/osf.io/ps2mh.

[7] Yousef, 2019. "Motion Induced Colours Fusion." PsyArXiv. doi:10.31234/osf.io/mkth2.

[8] Yousef, 2019. "Illusory Snakes Might Be Due to Asynchronized Respective Field Remapping." PsyArXiv. doi:10.31234/osf.io/ve4s8 [9] Yousef, 2019. "Idle Retinal Peripheries Diminish Spatial Wrapping." PsyArXiv. doi:10.31234/osf.io/srydk.

[10] Yousef, 2019. "Two Distinct Fusiform Face Areas." PsyArXiv. doi:10.31234/osf.io/a8gzv.

[11] Yousef, 2019. "Inactivated Peripheral Fusiform Face Area." PsyArXiv. doi:10.31234/osf.io/u 4xyz.

[12] Yousef, 2019. "Deep Breathing Alters Visual Motion Perception." PsyArXiv. doi:10.31234/osf.io/up3sa.

[13] Yousef, 2020. "Voluntary Hand Movements Discipline Audiovisual Perception.” PsyArXiv. doi:10.31234/osf.io/bqr6u. [14] Yousef, 2019. "Consciousness Might Be Localized in Extra Physical Dimensions.” PsyArXiv. doi:10.31234/osf.io/angc8. [15] Yousef, A. 2019. "But Where Is Consciousness." YouTube Link: https://www.youtube.com/watch?v=I1G3Jx-Q1YY

[16] Yousef, 2019. "Tachyons but Not Photons Might Generate Conscious Dreams." PsyArXiv. doi:10.31234/osf.io/hvqy7. [17] Yousef, 2019. "Voluntary Blinks Stop Binocular Rivalry." PsyArXiv. doi:10.31234/osf.io/z4jrn.

[18] Yousef, 2020. "Voluntary Movements Regulate Binocular Rivalry.” PsyArXiv. doi:10.31234/osf.io/qk249.

[19] Yousef, 2020. "Deep Breathing Governs Binocular Rivalry." PsyArXiv. doi:10.31234/osf.io/zr3uk.

[20] Yousef, 2019. "Motion Triggers Indefinite Stoppage Against Binocular Rivalry." PsyArXiv. doi:10.31234/osf.io/4qbrg.

[21] Yousef, 2019. "Linking Dynamics Between Pupil and Lens." engrXiv. doi:10.31224/osf.io/9c3vk.

[22] Yousef, 2021. "Voluntary Switching of Visual Motion Rivalry Estimate Human Heart Rate." PsyArXiv. doi:10.31234/osf.io/3ydqr. 